ISSN 1692-5858 | Vol. 18 \#1 | Enero -junio de 2020 | P. 11-22

\title{
Relación del uso del teléfono celular y los niveles de atención en el proceso de enseñanza - aprendizaje
}

\author{
Amparo Marina Vásconez Villavicencio \\ Pontificia Universidad Católica del Ecuador \\ flaka_amparo@hotmail.es \\ https://orcid.org/0000-0001-5151-6961 \\ Eleonor Virginia Pardo Paredes \\ Pontificia Universidad Católica del Ecuador \\ Av. Manuela Sáenz, Sector El Tropezón \\ epardo@pucesa.edu.ec \\ https://orcid.org/0000-0001-8068-9634
}

Paysandú y Potosí. Ciudadela La Victoria. Ambato. Ecuador
How to cite this paper:

Vásconez-Villavicencio, Amparo Marina; Pardo -Paredes, Eleonor Virginia (2020). Relación del uso del teléfono celular y los niveles de atención en el proceso de enseñanza - aprendizaje. Revista Encuentros, Universidad Autónoma del Caribe. Vol. 18-01.

Doi: 10.15665/encuent.v18i01.2168

Recibido: 19 de octubre de 2019 / Aceptado: 18 de diciembre de 2019

\section{RESUMEN}

El acceso a la telefonía móvil en Ecuador aumenta y la asistencia de estos dispositivos en niños y jóvenes está presente en sus actividades educativas; fundamento para analizar la relación del uso del teléfono celular con los niveles de atención en el proceso de enseñanza - aprendizaje en la Unidad Educativa Francisco Flor, Tungurahua, Ambato, mediante un diseño no experimental, transeccional, de campo. Se aplicó un cuestionario tipo encuesta a 431 estudiantes de bachillerato y la discusión de resultados permiten deducir que el uso del teléfono móvil con relación a los niveles de atención tiene una incidencia poco significativa, por lo que no interfiere en forma negativa, ni como elemento distractor del acto didáctico.

Palabras clave: teléfono celular, enseñanza, aprendizaje, atención.

\section{The relationship between mobile phone use and attention le- vels in teaching - learning process}

\begin{abstract}
As the access to mobile phones in Ecuador increases, so is the presence of these devices in the educational activities of children and young people. It is because of this, that it is essential to analyze the relationship between mobile phone usage and how it affects attention levels in the learning process. Through a non-experimental and transversal approach consisting of two variables, a survey was conducted among 431 high school students at the Francisco Flor School in Tungurahua, Ambato. The results show that the use of the mobile phone has an insignificant impact in attention levels, therefore not negatively interfering or distracting students during the learning process.
\end{abstract}

Keywords: cell phone, teaching, learning, attention 


\title{
A relação entre uso de telefones celulares e níveis de atenção no processo de ensino - aprendizagem
}

\begin{abstract}
RESUMO
À medida que o acesso a telefones celulares no Equador aumenta, aumenta também a presença desses dispositivos nas atividades educacionais de crianças e jovens. É por isso que é essencial analisar a relação entre o uso do telefone celular e como isso afeta os níveis de atenção no processo de aprendizagem. Por meio de uma abordagem não experimental e transversal, composta por duas variáveis, foi realizada uma pesquisa com 431 alunos do ensino médio da escola Francisco Flor em Tungurahua, Ambato. Os resultados mostram que o uso do telefone celular tem um impacto insignificante nos níveis de atenção, portanto, não interfere ou distrai negativamente os alunos durante o processo de aprendizagem.
\end{abstract}

Palavras-chave: telefone celular, ensino, aprendizado, atenção.

\section{Introducción}

En el mundo actual el desarrollo de las telecomunicaciones ha tenido un alto impacto en la población, permitiendo conocer los sucesos ocurridos a nivel nacional e internacional en tiempo real. Ejemplo de ello han sido los avances en la telefonía móvil, en la cual, los smartphones o teléfonos inteligentes permiten el fácil acceso a grandes cantidades de información, a través de la conexión a internet mediante redes inalámbricas de alta velocidad, el uso de diferentes aplicaciones, acceso a redes sociales, libros, revistas, videos, música, correo electrónico, chat, mensajería instantánea, entre otros, que hacen posible que la sociedad esté comunicándose en todo momento.

La utilización del teléfono móvil que cada día va en aumento puede catalogarse como el avance tecnológico másimportantedelosúltimosaños. EnEcuador,laAgencia de Regulación y Control de las Telecomunicaciones (ARCOTEL), publicó una infografía con datos sobre los abonados del Servicio Móvil Avanzado - SMA y de acceso a internet, correspondiente a junio del año 2017, donde existen 8,1 millones de cuentas de internet móvil y el 46,4\% de usuarios del SMA poseen un smartphone. La tenencia de internet móvil ha crecido desde 2010 en 46 puntos: de $2.4 \%$ a $48,7 \%$ y 11 de cada 100 niños, de entre 5 y 15 años, posee un teléfono móvil activo, y de ellos 6 usan un smartphone. (ARCOTEL, 2017, p.1)

Ahora bien, menciona el referido informe que:
La estadística de internet móvil refleja un incremento importante de las cuentas de este servicio desde 2010. Si consideramos los datos para el periodo 2015 2017, la tasa promedio de crecimiento en el servicio de internet móvil está directamente relacionada con la penetración del servicio móvil avanzado SMA en el Ecuador. Se considera que la implementación de tecnologías móviles tales como las de generación $3 \mathrm{G}$ y $4 \mathrm{G}$ por parte de los prestadores de SMA, ha facilitado el acceso a los usuarios a plataforma de navegación cuyo uso se encuentra incentivado por la oferta de contenidos y el acceso a las redes sociales.

Asimismo informa ARCOTEL, en su boletín estadístico de junio de 2018 que: "respecto de la población del país que tiene acceso al servicio móvil avanzado, el 89,54\% lo tiene" (ARCOTEL, 2018, p.1).

Como claramente se evidencia de esta información el acceso a la telefonía móvil en Ecuador va en aumento y en la cotidianidad se encuentra la asistencia de estos dispositivos electrónicos en los niños y jóvenes durante el cumplimiento de sus actividades educativas. La mayor parte de las horas del día esta población las dedica a estudiar y por ende a permanecer en sus aulas de clase, lugar donde llevan consigo su teléfono celular. Por lo antes expuesto cabe preguntarse, ¿Cuál es la relación del uso del teléfono celular con los niveles de atención en el proceso de enseñanza - aprendizaje?

Aun cuando el teléfono celular se considera un equipo de uso cotidiano que permite estar al tanto de 
las circunstancias de manera inmediata, de situaciones de urgencia y, lógicamente, tener una vida social más activa, la utilización del mismo, en el aula de clase, pudiera interferir en la atención de los alumnos, como un elemento distractor, por lo que los resultados de esta investigación generaron elementos para despejar la interrogante planteada.

\section{Fundamento teórico}

La producción científica que aborda el fenómeno del uso del teléfono celular se ha incrementado en las bases de datos especializadas en los últimos años; esto puede deberse a que es muy alta la población que cuenta con uno y que aparentemente es la forma más fácil y eficiente para comunicarse. El aula de clase no escapa de ello y menos su participación en el proceso de enseñanza-aprendizaje. Es así como Acero (2017) realizó un trabajo titulado "Análisis del uso del celular y su influencia negativa en el rendimiento académico de los estudiantes de los terceros años de bachillerato de la Unidad Educativa Colegio Nacional Galápagos”.

El mismo permitió identificar los factores de influencia de los celulares en la formación de los estudiantes para demostrar en qué medida afectan en el rendimiento académico. A través de una investigación bibliográficadocumental y método mixto aplicó 70 encuestas a estudiantes de los terceros años de bachillerato. Los resultados arrojaron que el $84 \%$ de los alumnos afirmaron llevar consigo el celular al aula de clase. El análisis permitió inferir que los estudiantes se encuentran inmersos en el uso del celular, tanto para realizar sus tareas académicas como para relacionarse con amigos y un $90 \%$ de los docentes coincidieron en que un alto porcentaje de estudiantes utilizan su celular dentro del aula con fines de entretenimiento, así como para establecer nuevas relaciones sociales, lo cual interfiere en sus actividades intelectuales, y por ende en su rendimiento académico.

Ahora bien, para reforzar el planteamiento hipotético se encuentra el trabajo hecho por Bernabéu (2017), intitulado: "La atención y la memoria como claves del proceso de aprendizaje”. A través de una revisión de los principales estudios y estrategias metodológicas que pueden aplicarse en las aulas, concluyó que:

los mecanismos atencionales y los mecanismos de memoria son las principales funciones neuropsicológicas que sostienen los procesos de aprendizaje. La aplicación de estrategias y metodologías adecuadas, basadas en el conocimiento de los sistemas neurales y procesos implicados en estas funciones permite favorecer y mejorar la adquisición de conocimientos en el entorno académico (Bernabéu, 2017, p.1)

Aguirre (2016) realizó una investigación para analizar la relación existente entre la atención y memoria, y el uso del teléfono inteligente en el interior del aula de clase. A través de un diseño ex-post facto, transversal y de tipo correlacional, aplicó los test de percepción de diferencias y de CARAs para evaluar la atención en los estudiantes, y las sub-pruebas de retención de dígitos en orden directo e inverso del WISC-IV para evaluar la memoria. Aplicó una encuesta para medir la percepción de los estudiantes respecto a la frecuencia del uso del teléfono inteligente en el aula y la percepción de la repercusión que tiene ese uso sobre la atención y la memoria, encontrando una relación estadísticamente significativa entre el impacto del uso del teléfono inteligente en clase y la memoria.

Con referencia a la atención encontró una relación directa leve, aunque estadísticamente no significativa. Los resultados permitieron proponer un programa para estimular la atención y memoria en los estudiantes.

En relación con el aprendizaje, Ojeda (2014) concluyó que "el uso de estrategias de aprendizaje es determinante para el rendimiento escolar", hallazgo encontrado en su investigación: Influencia de la atención y las estrategias de aprendizaje en el rendimiento académico. Las investigaciones sirvieron para abordar teóricamente los elementos de este estudio, a saber:

\section{La atención en el proceso de enseñanza- aprendizaje.}

Históricamente el estudio del término atención tiene sus orígenes desde el siglo pasado, se fundamenta, principalmente, en lo afirmado por WilliamJames, según el cual la atención:

No es solamente la capacidad mental para captar la mirada en uno o varios aspectos de la realidad y prescindir de los restantes, es el tomar posesión por parte de la mente, de forma clara y vívida, de uno de entre los varios posibles objetos de pensamiento que aparecen simultáneamente. Su esencia está constituida 
por focalización, concentración y conciencia. Atención significa dejar ciertas cosas para tratar efectivamente otras. (pp. 403-404).

Diferentes disciplinas y diversos enfoques teóricos se han dado al término atención. La misma ha sido analizada desde otras disciplinas y como proceso psicológico inmerso en el aprendizaje a partir de la psicología del desarrollo (Delgado, 2015). Para Ballesteros (2000) la atención es:

El proceso a través del cual podemos dirigir nuestros recursos mentales sobre algunos aspectos del medio, los más relevantes. Hace referencia al estado de observación y de alerta que nos permite tomar conciencia de lo que ocurre en nuestro entorno. Lo correlaciona con la conciencia (p.25)

Para Gazzaniga, Ivry \& Mangun (2014) la atención se define como:

la capacidad de enfocar la conciencia en un estímulo, pensamiento o acción mientras se ignora otro irrelevante. La atención influye en cómo procesamos las entradas sensoriales, almacenar esa información en la memoria, procesarla semánticamente y actuar en consecuencia (p.275).

De acuerdo con Ramos-Galarza, Paredes, Andrade, Santillán \& González (2016) la atención es una función neuropsicológica que posee subprocesos de focalización, selectividad, sostenibilidad, división y alternancia, que permiten al ser humano identificar un determinado estímulo para realizar un procesamiento cognitivo y cerebral (p.33). En atención a esto y según Portellano (2005) citado por Ramos-Galarza et al. (2016) "es un sistema funcional complejo, dinámico, multimodal y jerárquico que facilita el procesamiento de la información en sus diferentes componentes sensoriales, cognitivos o motores" (p.34).

Ahora bien, en el proceso de enseñanza-aprendizaje la atención juega un papel importante no como un elemento aislado del proceso cognitivo sino como complemento de las acciones mentales de observar, memorizar, aprehender, entre otras. Siguiendo a Ríos-Lago, MuñozCéspedes \& Paúl-Lapedriza (2007) es un conjunto de diferentes mecanismos coordinados que permite escoger del entorno los estímulos principales que sirven para llevar a cabo una acción y alcanzar unos objetivos.
Es así como las teorías del aprendizaje ponen énfasis en la conducta observable y en los estímulos ambientales y que, de acuerdo con Delgado (2015) se ubican en el paradigma conductista según el cual sólo se puede estudiar científicamente aquello que puede ser observado y medido directamente. Afirman los conductistas que el desarrollo es función del aprendizaje, de las experiencias vividas en el entorno. Las tres versiones de este enfoque son el condicionamiento clásico de Pavlov, el condicionamiento operante de Skinner y la teoría cognitivo-social de Bandura (p. 276-278). El aprendizaje se convierte en un proceso cognitivo a partir de experiencias y sensaciones afectadas por el ambiente exterior donde impera la capacidad de seleccionar y comprender información como características fundamentales de la atención.

Londoño (2009) expone que de acuerdo con el modelo clínico propuesto por Sohlberg \& Mateer (1987 y 1989) se definen algunos elementos constitutivos del proceso de atención, los cuales participan de forma activa e interna en el procesamiento de la información de cualquier modalidad sensorial, a saber: arousal, atención focalizada, atención sostenida, atención selectiva, atención alternante y dividida. De acuerdo con RíosLago et al. (2007), el modelo:

Más que una explicación del funcionamiento real de los procesos atencionales, este modelo ha mostrado una utilidad heurística y describe con claridad aspectos fenomenológicos de los procesos atencionales. Es jerárquico y asume que cada nivel atencional requiere el funcionamiento correcto del nivel anterior (p.291).

A continuación se presenta el cuadro que incluye los diferentes niveles de atención del modelo clínico de Sohlberg \& Mateer (1987 y 1989). 


\begin{tabular}{|c|c|}
\hline Arousal & $\begin{array}{l}\text { Capacidad de estar despierto y de mantener la alerta. Implica la capacidad de } \\
\text { seguir estimulos u ordenes. Es la activacion general del organismo. }\end{array}$ \\
\hline Atención focal & $\begin{array}{l}\text { Habilidad para enfocar la atencion a un estimulo determinado. No se valora } \\
\text { el tiempo de fijacion al estímulo. Se suele recuperar en las fases iniciales tras } \\
\text { un traumatismo craneoencefalico. Al principio puede responderse } \\
\text { exclusivamente a estimulos internos (dolor, temperatura, etc). }\end{array}$ \\
\hline Atención sostenida & $\begin{array}{l}\text { Capacidad de mantener una respuesta de forma consistente durante un } \\
\text { periodo de tiempo prolongado. Se divide en dos subcomponentes: 1) se } \\
\text { habla de vigilancia cuando la tarea es de detección y de concentración } \\
\text { cuando se refiere a otras tareas cognitivas, y 2) nocion de control mental o } \\
\text { memoria operativa, en tareas que implican el mantenimiento y maripulación } \\
\text { de información de forma activa en la mente. }\end{array}$ \\
\hline Atención selectiva & $\begin{array}{l}\text { Capacidad para seleccionar, de entre varias posibles, la información relevante } \\
\text { a procesar o el esquema de acción apropiado, inhibiendo la atención a unos } \\
\text { estimulos mientras se atiende a otros. Los pacientes con alteraciones en este } \\
\text { nivel sufren numerosas distracciones, ya sea por estimulos externos o } \\
\text { internos. }\end{array}$ \\
\hline Atención alternante & $\begin{array}{l}\text { Capacidad que permite cambiar el foco de atención entre tareas que implican } \\
\text { requerimientos cognitivos diferentes, controlando que información es } \\
\text { procesada en cada momento. Las alteraciones de este nivel impiden al } \\
\text { paciente cambiar rápidamente y de forma fluida entre tareas. }\end{array}$ \\
\hline Atención dividida & $\begin{array}{l}\text { Capacidad para atender a dos cosas al mismo tiempa. Es la capacidad de } \\
\text { realizar la seleccion de más de una informacion a la vez o de más de un } \\
\text { proceso o esquema de accion simultáneamente. Es el proceso que permite } \\
\text { distribuir los recursos atencionales a diferentes tareas o requisitos de una } \\
\text { misma tarea. Puede requerir el cambio rápido entre tareas o la ejecucion de } \\
\text { forma automatica de alguna de ellas. }\end{array}$ \\
\hline
\end{tabular}

Tomado de: Sohlberg \& Mateer (1987 y 1989) citado por Ríos-Lago et al (2007)

En este orden de ideas y para el desarrollo del objetivo de esta investigación se asume la definición de la atención dividida del mencionado modelo clínico como:

La capacidad para atender dos cosas al mismo tiempo. Es la capacidad de realizar la selección de más de una información a la vez o de más de un proceso o esquema de acción simultáneamente. Es el proceso que permite distribuir los recursos atencionales a diferentes tareas o requisitos de una misma tarea. Puede requerir el cambio rápido entre tareas o la ejecución de forma automática de alguna de ellas.

De acuerdo con Villaroig y Muiños (2017, p.16) para lograr la selección debe darse una oscilación de la atención que permita desplazar la atención de una información a otra de forma rápida para no perder la recepción y procesamiento de estímulos relevantes. Para que esta estrategia sea eficaz, aquellas tareas que se deben atender, han de pertenecer a la misma modalidad sensorial.

En correspondencia con lo planteado por Villaroig y Muiños (2017), en la definición de atención dividida de Sohlberg \& Mateer (1987 y 1989) destaca como elemento preponderante la capacidad de seleccionar información necesaria, lo que es aplicable en la ejecución del proceso de enseñanza-aprendizaje. Por ello, según Nicoletti (2006) la acción de aprender se compone de:

Una secuencia encaminada a la construcción del conocimiento, desarrollo de habilidades, adquisición de hábitos y formación de actitudes, originando una transformación en la conducta del alumno. Los procesos cognitivos, como el razonamiento, la memoria, la concentración y la atención, se corresponden con todas las acciones y procesos internos que realiza la persona cuando está 
organizando sus estructuras en función de alcanzar una asimilación significativa (p.13).

Al respecto, Díaz y Hernández (1997) expresan que el proceso de enseñanza debería orientarse a aculturar a los estudiantes por medio de prácticas auténticas (cotidianas, significativas, relevantes en su cultura), apoyadas en procesos de interacción social similares al aprendizaje artesanal. En gran medida se plasman aquí las ideas de la corriente sociocultural vigotskyana, en especial la provisión de un andamiaje de parte del profesor (experto) hacia al alumno (novato), que se traduce en una negociación mutua de significados.

Por ello, de acuerdo con los mencionados autores es en el proceso de enseñanza, donde la actuación del docente se convierte en el acto didáctico que pasa por propiciar un ambiente donde el estudiante vierta toda su capacidad de atención con la intención de aprender. Es en el acto didáctico, también, donde el docente incorpora elementos prácticos y reales para enseñar y donde el uso de tecnologías de información y comunicación (TIC's), como estrategias innovadoras, constituyen herramientas actuales para fortalecer la enseñanza e incentivar el aprendizaje. Es en la atención dividida donde el alumno puede observar, aprehender, comprender y procesar selectivamente la información generada en el proceso educativo.

\section{El teléfono celular como elemento de las tecnologías de información y comunicación en la enseñanza}

En la actualidad el uso de las TIC's avanza vertiginosamente sobretodo en la población más joven y como elemento complementario de la enseñanza. En este sentido se definen como:

Conjunto de tecnologías existentes que hacen posible elaborar, reunir, almacenar y modificar todo tipo de información, a la vez que permiten registrar y presentar dicha información a través de imágenes y sonidos en múltiples formatos; esto hace posible que la información existente en el mundo antes en los libros o en otras formas físicas sea digitalizada adquiriendo como característica la inmaterialidad y haciendo posible la existencia de una realidad virtual (Aguirre, 2016, p.22)
En la enseñanza Ramírez-Montoya y García-Peñalvo (2017) consideran que el dispositivo móvil tiende a modificar las prácticas educativas por aportar elementos de independencia personalizados que otras tecnologías no ofrecían anteriormente.

Los teléfonos celulares cuentan con los elementos característicos de la definición de Aguirre (2016). Tienen la capacidad principal de recopilar y modificar datos y poseen un infinito campo de opciones que pueden ser efectivas y eficientes para los estudiantes durante sus clases, tales como: internet, toma de fotografías de alta definición, trasferencia de archivos, comunicación por video en tiempo real, entre otras.

Es así como el uso del teléfono celular pasó de ser un mero instrumento de comunicación a convertirse en una memoria portátil. Según Malo (2006) el teléfono móvil ha sido una de las nuevas tecnologías mejor aceptadas entre los jóvenes expandiendo su uso y disposición de manera veloz. Pero, si bien ha sido una incorporación positiva como producto innovador en materia de telecomunicaciones, su uso ha llenado todos los espacios de la vida de muchas personas.

Es así como en el aula, concebido naturalmente como un punto de encuentro para enseñar y aprender, el teléfono celular ha llegado a incorporarse como un elemento casi cotidiano y casi universalmente usado por los estudiantes en su día a día. Al respecto Navarrete y Mendieta (2018) consideran que en el aula de clases, las TIC son potenciales instrumentos cognitivos que sirven para ampliar las capacidades intelectuales y estimular el desarrollo de competencias como investigar, seleccionar, organizar y manejar nueva información necesarias en la acción de aprender.

De acuerdo con la Organización de las Naciones Unidas para la educación, la ciencia y la cultura (UNESCO, 2013) "aprender" ya no consiste en adquirir y memorizar un conjunto de contenidos predefinidos, sino en saber crear, gestionar y comunicar el conocimiento en colaboración con otros.

Ahora bien, es completamente plausible pensar que el uso del teléfono celular en el aula de clase pueda estar asociado a dificultar el proceso de enseñanzaaprendizaje, toda vez que pudiera convertirse en un elemento distractor para los alumnos. Lo evidente es el uso masivo del teléfono celular que, dentro del campo 
de las TIC's, resaltan y destacan como instrumentos útiles para comunicarse y obtener información rápida y veraz, modificando también la forma cotidiana de interrelacionarse las personas.

Lasica (2007) afirma que las tecnologías móviles innovadoras están causando disrupción, cambios inalterables a la forma en que la próxima generación, trabaja, juega e interactúa con el resto del mundo. Incluso más, algunos identifican el teléfono móvil como el "séptimo medio" (Ahonen, 2008), ya que además de reproducir todos los medios de comunicación tradicionales presenta 5 elementos únicos: su carácter personal, disposición y acceso 24 horas al día, estar al alcance de la mano, posibilidad de consumo y ser un instrumento que permite canalizar el impulso creativo a través de aplicaciones multimedia, como la cámara de fotos, la grabadora de video y de voz.

Estas características hacen que el teléfono celular se convierta en un instrumento casi obligatorio del día a día y de esto no están excluidos los alumnos, en todo caso, la alta capacidad de adaptación a la tecnología que poseen los niños y jóvenes, lo hacen un objeto de uso diario que puede, desde la exterioridad, convertirse en un estímulo para llamar la atención de esos usuarios.

\section{Metodología}

El trabajo investigativo se fundamentó en una observación documental pues se analizó una realidad objetiva a través de la descripción de los elementos inmersos en el estudio. El diseño de la investigación fue no experimental, transeccional, de campo y univariable. Según Hernández et al (2014, p.154) el propósito de "los diseños de investigación transeccional o transversal es describir variables y analizar su incidencia e interrelación en un momento dado”. En función a ello, en esta investigación, los datos fueron recolectados en una sola oportunidad para lo cual se aplicó como técnica un cuestionario tipo encuesta a la población objeto de estudio constituida por 431 estudiantes de bachillerato de la Unidad Educativa Francisco Flor, ubicada en el Cantón Ambato, Provincia de Tungurahua, Ecuador, distribuida de la siguiente manera:
Tabla No. 1. Distribución de la población

\begin{tabular}{lccc}
\hline Año de Bachillerato & Matutino & Vespertino & Total \\
\hline Primer año & 80 & 92 & 172 \\
Segundo año & 57 & 79 & 136 \\
Tercer año & 54 & 69 & 123 \\
TOTAL & 191 & 240 & 431 \\
\hline \multicolumn{4}{l}{ Fuente: Elaboración propia (2019). }
\end{tabular}

Por cuanto la población fue finita, lo que permitió el acceso a todos los integrantes de la misma y por ende a la totalidad de los sujetos, se aplicó un censo poblacional. El instrumento de medición contó con 23 items en escala tipo Likert de 3 alternativas de respuesta, construido de acuerdo con los indicadores de la variable: niveles de atención. Asimismo, se fundamentó en el sistema teórico y fue sometido a validación a través de juicio de expertos en el área de psicología y pedagogía.

La confiabilidad del instrumento se determinó utilizando la fórmula del Alpha de Cronbach, luego de haberse aplicado una prueba piloto para constatar si la aplicación repetida del mismo a los individuos produce los mismos resultados; es decir, se mide la consistencia de los resultados al ser aplicado de manera repetida, tal como lo afirma Hernández et al. (2014, p.200), "la confiabilidad de un instrumento de medición es el grado en que su aplicación repetida al mismo individuo u objeto producen resultados iguales".

El tratamiento estadístico de los datos se realizó a través de la estadística descriptiva (media, desviaciones estándar, distribuciones de frecuencia, tablas y gráficos), utilizando el paquete estadístico SPSS, versión 20. Para el análisis de resultados se utilizó un baremo de acuerdo a las alternativas de respuesta, el cual se estructuró de la siguiente manera:

Tabla No. 2 Baremo para analizar los resultados

\begin{tabular}{cc}
\hline Valor & Ponderación \\
\hline 1 a 1,66 & Bajo \\
1,67 a 2,33 & Moderado \\
2,34 a 3,00 & Alto \\
\hline
\end{tabular}

\section{Resultados y discusión}

Los resultados se muestran de acuerdo con los indicadores, dimensiones y variable objeto de estudio, a saber: 
Acceso a información de contenidos de la clase: Un 96,8\% de los encuestados respondieron que nunca tienen acceso a la información de los contenidos de aprendizaje en clase, a través de su teléfono celular; mientras que el 3,2\% indicó que a veces sí tienen acceso a esta información. Los datos se reflejan en la tabla No. 3 y el gráfico No. 1, respectivamente. En contraposición con lo afirmado por Hernández (2017) y UNESCO (2013) el proceso de enseñanza-aprendizaje, en este caso, no se beneficia con el uso de herramientas tecnológicas como el teléfono celular, cuyo auge se ha globalizado como necesario para aprender, aprehender y comprender contenidos educativos.

Tabla No. 3. Acceso a información de contenidos

\begin{tabular}{lcccc}
\hline Válido & Frecuencia & Por ciento & \% Válido & \% Acumulado \\
\hline Nunca & 417 & 96,8 & 96,8 & 96,8 \\
Aveces & 14 & 3,2 & 3,2 & 100,0 \\
Total & 431 & 100,0 & 100,0 & \\
\hline
\end{tabular}

Fuente: Elaboración propia (2019).

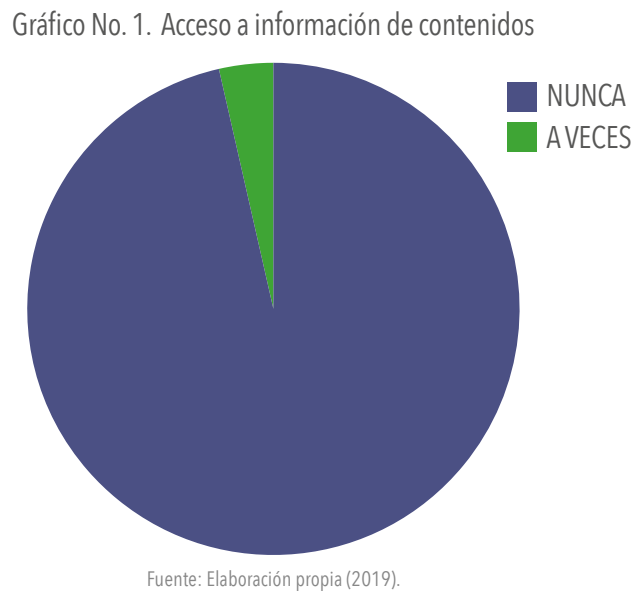

Ahora bien, según se indica en la tabla No. 4, el resultado de la estadística descriptiva (media aritmética 1,03 y 0,177 desviación estándar) demuestra que existe un bajo uso del teléfono celular en el aula de clase, de lo que se puede inferir que, en este caso, los alumnos tienden a utilizar poco el teléfono celular como un instrumento para reforzar el aprendizaje de los contenidos educativos.

Las causas de este poco uso pudieran estar asociadas a que el docente completa exitosamente la explicación o bien, que los alumnos no tienen interés en realizar esta búsqueda, aun cuando Malo (2006) afirma que el teléfono móvil ha sido una de las nuevas tecnologías mejor aceptadas entre los jóvenes cuyo uso se ha ido expandiendo de forma veloz.

\begin{tabular}{cc} 
Tabla No. 4. Estadísticas Acceso a información de contenidos \\
\hline Válido & 431 \\
\hline Perdido & 0 \\
Media & 1,0325 \\
Desviación estándar &, 17748
\end{tabular}

Fuente: Elaboración propia (2019).

De acuerdo con Campión, Diez y Navaridas (2014) el uso de TIC's en educación requiere la implementación de estrategias de aprendizaje que permitan al estudiante en forma autónoma construir su propio conocimiento, mediante una gestión de la clase caracterizada por periodos cortos, interactivos, multidisciplinares, enfoques globales, amenos, flexibles e interesantes.

Aplicación en la resolución de problemas: En este indicador se puede observar que el 68,9\% de los alumnos nunca usan el teléfono celular para resolver problemas en clase mientras que el $31,1 \%$, a veces si lo utilizan, tal como se aprecia en la tabla No.5 y el gráfico No. 2.

Tabla No. 5. Aplicación en la resolución de problemas

\begin{tabular}{lcccc}
\hline Válido & Frecuencia & Por ciento & \% Válido & \% Acumulado \\
\hline Nunca & 297 & 68,9 & 68,9 & 68,9 \\
A veces & 134 & 31,1 & 31,1 & 100,0 \\
Total & 431 & 100,0 & 100,0 & \\
\hline \multicolumn{5}{c}{ Fuente: Elaboración propia (2019). }
\end{tabular}

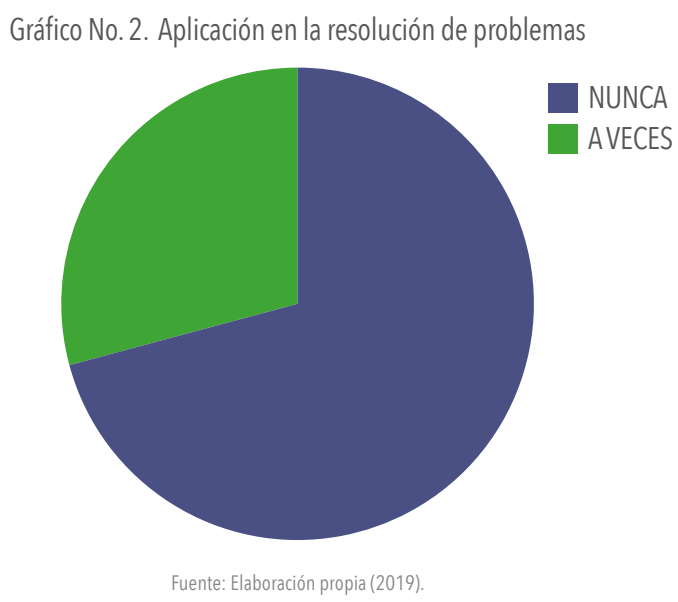


En este sentido se muestra en la tabla No. 6 los resultados de la estadística descriptiva: una media aritmética de 1,31 y desviación estándar de 0,46 que indican una tendencia a un bajo uso del teléfono celular en la resolución de problemas en el aula de clase.

\begin{tabular}{cc} 
Tabla No. 6. Estadísticas Aplicación en la resolución de problemas \\
\hline Válido & 431 \\
\hline Perdido & 0 \\
Media & 1,3109 \\
Desviación estándar &, 46340 \\
\hline \multicolumn{2}{c}{ Fuente: Elaboración propia (2019). }
\end{tabular}

En este caso se aprecia nuevamente que los alumnos tienen poco interés en usar el teléfono celular como herramienta tecnológica para facilitar el proceso de enseñanza-aprendizaje; contrario a lo afirmado por Ojeda (2014) cuyo resultado investigativo le permitió afirmar que "el uso de estrategias de aprendizaje es determinante para el rendimiento escolar"; asumiendo que dicho dispositivo cuenta con aplicaciones prácticas y accesibles a cualquier nivel de desarrollo cognitivo.

Según Campión, Diez y Navaridas (2014) quienes hacen un análisis de los niveles propuestos por Bloom en el proceso cognitivo, aplicar, refiere a hacer uso de conocimiento nuevo (adquirido) para resolver problemas de un modo diferente. Este nivel de conocimiento requiere contar con herramientas y formas didácticas que le permitan al alumno acceder en tiempo real a solucionar actividades formativas en el aula de clase, lo que sucede en forma baja en el grupo de encuestados.

Uso de internet y TICS: Para conocer si los alumnos utilizan el teléfono celular y acceder a internet u otras tecnologías de información y comunicación se formularon preguntas relacionadas con el uso de chat, videos y variadas formas de comunicarse. Al respecto el 5,6\% afirmó que nunca usan el teléfono celular para estas acciones en clase, mientras que el 42,9\% indicó que a veces y finalmente el 51,7\% siempre lo emplea. Los datos mencionados se reflejan en la tabla No. 7 y el gráfico No. 3.
Tabla No. 7. Uso de internet y TICS

\begin{tabular}{lcccc}
\hline Válido & Frecuencia & Por ciento & \% Válido & \% Acumulado \\
\hline Nunca & 24 & 5,6 & 5,6 & 5,6 \\
A veces & 184 & 42,7 & 42,7 & 48,3 \\
Siempre & 223 & 51,7 & 51,7 & 100,0 \\
Total & 431 & 100,0 & 100,0 & \\
\hline \multicolumn{5}{c}{ Fuente: Elaboración propia (2019). }
\end{tabular}

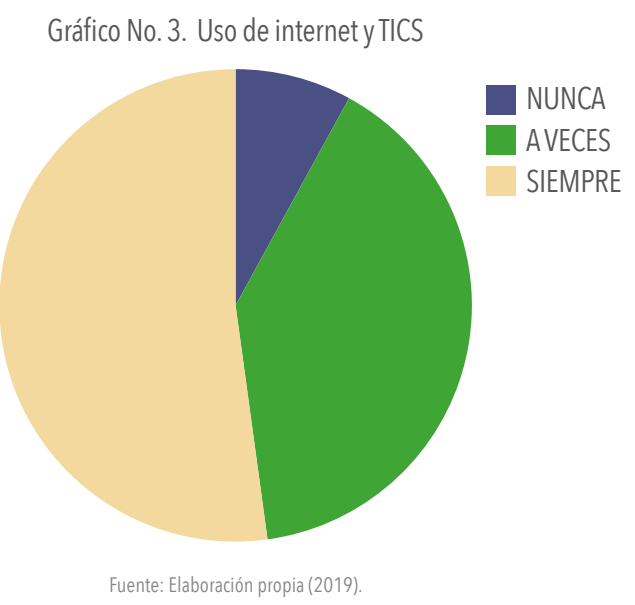

Para este indicador la estadística descriptiva (tabla No. 8) arrojó un promedio de 2,46 y una desviación estándar de 2,60; por lo cual la tendencia del uso del teléfono celular es alto con referencia a la utilización del chat para compartir información en clase, elaborar exposiciones y/o para comunicarse entre ellos. Pareciera que aun cuando no lo utilizan para ampliar el contenido de la clase ni para hacer los deberes in situ, sí lo emplean para entablar conversaciones que, en algunos casos, pudiera incorporar algún dato académico aunque no necesariamente. En todo caso, los alumnos respondieron que lo utilizan como dispositivo de comunicación, tal como lo afirman Lasica (2007) y Ahonen (2008) al caracterizarlos como dispositivos de comunicación e interrelación entre los jóvenes por su versatilidad y diversidad al procesar, acceder y transmitir información.

\begin{tabular}{cc} 
Tabla No. 8. Estadísticas Uso de internet y TICS \\
\hline Válido & 431 \\
\hline Perdido & 0 \\
Media & 2,4617 \\
Desviación estándar &, 60062 \\
\hline
\end{tabular}

Fuente: Elaboración propia (2019). 
Tal como refieren Bernal y Hernández (2015) la tecnología forma parte de los métodos de enseñanza y se convierte en una herramienta didáctica; así como también permite la interrelación con el entorno, logrando una mejora en la calidad del proceso enseñanzaaprendizaje. Al respecto, el informe de la UNESCO (2013) menciona las posibilidades únicas que ofrecen los dispositivos móviles; vistos como un complemento de las inversiones en educación, entre las que destacan: facilitar el aprendizaje personalizado; habilitar el aprendizaje en cualquier momento y en cualquier lugar; asegurar un uso más productivo del tiempo en clase (flipped classroom); crear nuevas comunidades de aprendizaje y facilitar la continuidad de la experiencia de aprendizaje.

Atención dividida: La tabla No. 9 y el gráfico No. 4 muestran los resultados de este indicador, donde el $100 \%$ de los alumnos indicaron que a veces pueden realizar dos actividades de manera simultánea en clase, reforzando las características del concepto teórico asumido en esta investigación de acuerdo con los autores Sohlberg \& Mateer.

Tabla No. 9. Atención dividida

\begin{tabular}{lcccc}
\hline Válido & Frecuencia & Por ciento & \% Válido & \% Acumulado \\
\hline A veces & 431 & 100,0 & 100,0 & 100,0 \\
\hline \multicolumn{5}{c}{ Fuente: Elaboración propia (2019). }
\end{tabular}

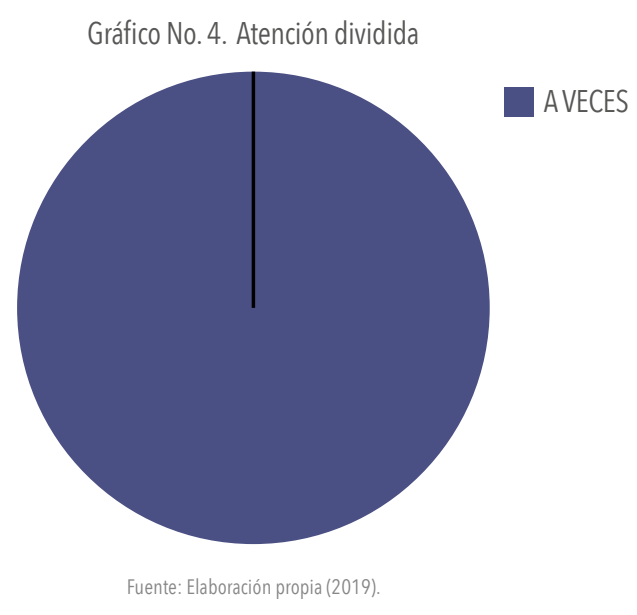

Para afianzar lo planteado anteriormente, los resultados de la estadística descriptiva para este indicador muestran que la atención dividida de los estudiantes tiende a ser moderada es decir, mediamente pueden realizar dos acciones simultáneas o bien pueden escoger entre dos actividades para atender.
Tabla No. 10. Estadísticas Atención dividida

\begin{tabular}{cc}
\hline Válido & 431 \\
\hline Perdido & 0 \\
Media & 2,0000 \\
Desviación estándar &, 00000 \\
\hline \multicolumn{2}{c}{ Fuente: Elaboración propia (2019). }
\end{tabular}

De acuerdo con Viramonte (2000, p.30) citado por Fuenmayor y Villasmil (2008) los procesos cognitivos básicos son aquellos que como la percepción, la atención y la memoria, se pueden producir sin la intervención consciente del sujeto y tienen una raíz biológica; no obstante, lo anterior no implica que el sujeto no pueda, ulteriormente, llegar a algún grado de control e intencionalidad en su realización.

Al respecto refieren Fuenmayor y Villasmil (2008, p.193) que la persona adquiere destrezas y desarrolla rutinas automáticas que le permiten realizar una serie de tareas sin prestar, según parece, mucha atención. Para ello el docente deberá estar en capacidad de utilizar estrategias didácticas que conlleven a propiciar un ambiente educativo idóneo donde el alumno pueda experimentar, por sí mismo situaciones que conlleven a fortalecer está capacidad atencional.

Impacto del uso del celular con los niveles de atención: Elresultadodelanálisis de todoslosindicadores para medir el impacto del uso del teléfono celular con los niveles de atención indica que es moderado, de acuerdo con el $60,1 \%$ y bajo que corresponde a un $39,9 \%$. La estadística descriptiva corrobora esta afirmación pues arrojó un promedio de 1,60 y una desviación estándar de 0,49 que permite apreciar una tendencia a la baja. Los datos se muestran en las tablas Nos. 11 y 12, gráfico No. 5 , respectivamente.

Tabla No. 11. Impacto del uso del celular con los niveles de atención

\begin{tabular}{lcccc}
\hline Válido & Frecuencia & Por ciento & \% Válido & \% Acumulado \\
\hline Bajo & 172 & 39,9 & 39,9 & 39,9 \\
Moderado & 259 & 60,1 & 60,1 & 100,0 \\
Total & 431 & 100,0 & 100,0 & \\
\hline \multicolumn{5}{c}{ Fuente: Elaboración propia (2019). }
\end{tabular}


Gráfico No. 5. Impacto del uso del celular con los niveles de atención

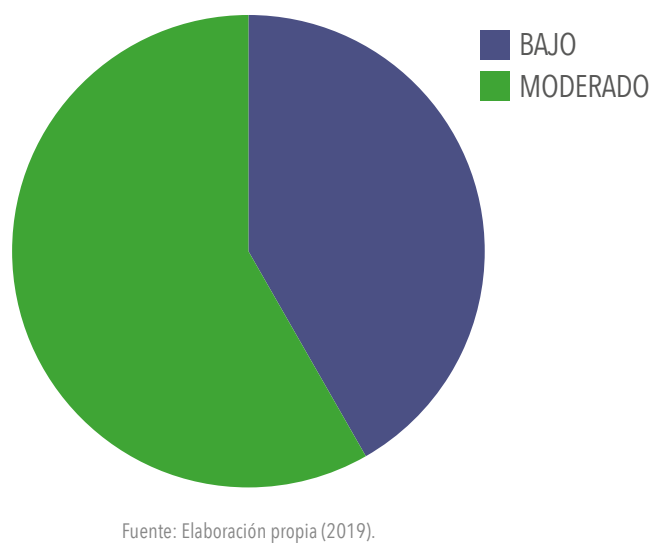

Tabla No. 12. Estadísticas Impacto del uso del celular con los niveles de atención

\begin{tabular}{cc}
\hline Válido & 431 \\
\hline Perdido & 0 \\
Media & 1,6009 \\
Desviación estándar &, 49028 \\
\hline
\end{tabular}

Fuente: Elaboración propia (2019).

El análisis de estos datos permiten deducir que el impacto del uso del teléfono celular con relación a los niveles de atención en el proceso de enseñanza-aprendizaje tiene una incidencia poco significativa, por lo que no interfiere en forma negativa ni como elemento distractor del acto didáctico; en todo caso pareciera que el manejo adecuado del teléfono celular por parte de los alumnos en clase podría servir como herramienta tecnológica que contribuya a una mayor participación del alumno. Los resultados corroboran la investigación realizada por Aguilar (2016) que demostró una relación directa leve, aunque estadísticamente no significativa entre el uso del teléfono celular en el aula de clase y la atención.

En el proceso de enseñanza-aprendizaje afirman Bernal y Hernández (2015) que el maestro deberá transformar en sus alumnos la utilidad que le han dado a sus teléfonos a una de perspectiva pedagógica y beneficiarse formativamente de su uso.

\section{Conclusiones y recomendaciones}

El estudio empírico permite afirmar que los alumnos utilizan el teléfono celular en clase, algunas veces para compartir información y para apoyar en la realización de sus deberes; sin embargo, el mismo tiene poca interferencia en el nivel de atención de los estudiantes y puede afirmarse que no es un elemento distractor en el proceso de enseñanza-aprendizaje, pues el uso del teléfono celular con relación a los niveles de atención en el proceso de enseñanza-aprendizaje resultó con una incidencia poco significativa.

Con respecto a la capacidad de atención, los datos aportados por los estudiantes encuestados permitieron inferir que los mismos tienen la capacidad moderada de realizar dos acciones simultáneas (utilizar el teléfono celular y atender a la clase), elementos del concepto de atención dividida asumido en este estudio y propuesto por Sohlberg \& Mateer (1987 y 1989).

Para el proceso de enseñanza-aprendizaje cabe destacar el bajo uso del teléfono celular como herramienta tecnológica para acceder a información y resolver problemas en clase, lo que se contrapone con los beneficios derivados de la aplicación de nuevas tecnologías necesarias para fortalecer el proceso educativo en el mundo de hoy, aun cuando sí es empleado cotidianamente por los alumnos para comunicarse.

La discusión de los resultados permitió construir las siguientes acciones:

- Propiciar el uso del teléfono móvil, en el aula de clase, como dispositivo electrónico avanzado para orientar la búsqueda de información educativa que permita reforzar el contenido de aprendizaje, con restricciones necesarias para que no interfiera con la comprensión de contenidos.

- Capacitar al docente en aplicaciones tecnológicas que puedan accederse a través del teléfono celular y que contengan material idóneo para utilizar en clase.

- Fortalecer en los alumnos la capacidad de atención dividida, a través de talleres de formación y estimulación, por cuanto la misma les servirá para su proceso educativo y para la toma de decisiones de vida.

- Consolidar la construcción del trabajo en equipo entre alumno y docente con el propósito de convertir el proceso de enseñanza-aprendizaje en un acto colaborativo, armonioso, participativo e innovador. 


\section{Referencias Bibliográficas}

Acero, 1. (2017). Análisis del uso del celular y su influencia negativa en el rendimiento académico de los estudiantes de los terceros años de bachillerato de la Unidad Educativa Colegio Nacional Galápagos. Informe Final de Investigación. Universidad Central del Ecuador. Ecuador. Recuperado de: $\quad$ http://www.dspace.uce.edu.ec/bitstream/25000/15079/1/T-UCE-0019IN003-2018.pdf

Aguirre, F. (2016) Relación del uso del teléfono inteligente en el aula de clase con la atención y lamemoria. Trabajo fin de master en neurospicología y educación. Universidad Internacional de la Rioja. Recuperado de: https://reunir. unir.net/bitstream/handle/123456789/4554/AGUIRRE\%20TELLEZ\%2C\%20 FABIO $\% 20$ ANDRES.pdf? sequence $=1$ \&isAllowed $=\mathrm{y}$

Ahonen, T. (2008) "Mobile as 7th of the Mass Media, cellphone, cameraphone, iPhone, smartphone", hardcover futuretext. Recuperado de: http://siteresources.worldbank.org/EXTEDEVELOPMENT/Resources/ TomiAhonenMobile7thMassMediaExcerpt.pdf

Ballesteros, J. y Reales, A. (2000). Atención y memoria. Recuperado de: http:// dialnet.unirioja.es/servlet/extaut?codigo $=528799$

Bernabéu, E. (2017). La atención y la memoria como claves del proceso de aprendizaje. Aplicaciones para el entorno escolar. Revista ReiDoCrea, 6(2), 1623. Recuperado de: https://www.ugr.es/ reidocrea/

Bernal, V., y Hernández, N. (2015).Aula móvil en el nivel bachillerato. Revista Perspectivas docentes volumen 58.TEXTOS Y CONTEXTOS. Recuperado de: http://ri.ujat.mx/bitstream/20.500.12107/2647/1/1198-973-A.pdf

Campión, R. Diez, A., Navaridas, F. (2014). La taxonomía del aprendizaje a debate: Del Modelo de Bloom de los años 50 a_la era del aprendizaje móvil. Universidad de La Rioja https://www.researchgate.net/publication/300003962

Delgado, M. (2015). Fundamentos de Psicología. Editorial Médica Panamericana

Díaz, F., Hernández, G. (1997). Estrategias para un aprendizaje significativo. Editorial Trillas. México.

Fuenmayor, G., Villasmil, Y. (2008). La percepción, la atención y la memoria como procesos cognitivos utilizados para la comprensión textual. Revista de Artes y Humanidades UNICA, vol. 9, núm. 22, mayo-agosto, 2008, pp. 187-202

Universidad Católica Cecilio Acosta. Maracaibo, Venezuela. Recuperado de: http://www.redalyc.org/articulo.oa?id=170118859011

Gazzaniga, M., Ivry , R., Mangun, R. (2014). Cognitive Neuroscience. The biology of the mind. Library of Congress Cataloging-in-publication data. Fourth edition. Recuperado de: http://docshare04.docshare.tips/files/29847/298473401. pdf

Gobierno de la República del Ecuador. (2017). Agencia de Regulación y Control de las Telecomunicaciones. Informe Estadístico. Infografía. Recuperado de: http:// www.arcotel.gob.ec.

Gobierno de la República del Ecuador. (2018). Agencia de Regulación y Control de las Telecomunicaciones. Boletín Estadístico. Junio. Recuperado de: http:// www.arcotel.gob.ec

Hernández, R., Fernández, C. y Baptista, P. (2014) Metodología de la Investigación, 6a . Edición, McGraw Hill/Interamericana Editores S.A DE C.V., México.

James, W. (1890). The principles of psychology. New York: Henry Holt and Company. Recuperado de: https://archive.org/details/theprinciplesofp0 l jameuoft

Lasica, D. (2007). "The Mobile Generation: Global Transformations at the Cellular Level: A report of the Fifteenth Annual Aspen Institute Roundtable on Information Technology. Washington, D.C.: Aspen Institute.

Londoño, L. (2009). La atención: un proceso psicológico básico. Recuperado de: https:/www.researchgate.net/publication/297760912

Malo, S. (2006). Impacto del teléfono móvil en la vida de los adolescentes entre 12 y 16 años. Revista científica de comunicación y educación No. 27. Huelva. España. Recuperado de: https://www.redalyc.org/pdf/158/15802716.pdf

Navarrete, G., Mendieta., C. (2018). Las TIC y la educación ecuatoriana en tiempos de internet: Breve análisis. Espirales revista multidisciplinaria de investigación. Vol. 2 No. 15. Abril. Recuperado de: DOI: https://doi.org/10.31876/re.v2i15.220

Nicoletti, J. (2006). Fundamento y construcción del Acto Educativo. Docencia e Investigación: revista de la Escuela Universitaria de Magisterio de Toledo. España. 31(16), 257-277. Recuperado de: https://ruidera.uclm.es/ xmlui/bitstream/handle/10578/8065/Fundamento y construcci n del Acto Educativo .pdf:sequence $=1$

Ojeda (2014). Influencia de la atención y las estrategias de aprendizaje en el rendimiento académico. Trabajo fin de master en neuropsicología y educación. Universidad Internacional de la Rioja. Recuperado de: https://reunir.unir.net/bitstream/handle/123456789/2396/ojeda\%20ojeda. pdf? sequence $=1$ \&isAllowed $=\mathrm{V}$

Ramírez-Montoya, M., García-Peñalvo, J. (2017), La integración efectiva del dispositivo móvil en la educación y en el aprendizaje. RIED. Revista Iberoamericana de Educación a Distancia (2017), 20(2), pp. 29-47.Recuperado de: DOI: http://dx.doi.org/10.5944/ried.20.2.18884

Ramos-Galarza, Paredes, L., Andrade, S., Santillán, W., González., L. (2016). Sistemas de Atención Focalizada, Sostenida y Selectiva en Universitarios de Quito-Ecuador. Revista Ecuatoriana de Neurología. Vol. 25, No.1-3. Recuperado de: http://revecuatneurol.com/wp-content/uploads/2017/05/Sistemas-atencionfocalizada-sostenida-selectiva-universitarios-quito-ecuador.pdf

Ríos-Lago, M., Muñoz, J. y Paúl, N. (2007). Alteraciones de la atención tras daño cerebral traumático: evaluación y rehabilitación. Revista Neurol, 44(5), 291-297. Recuperado de: http: http://www.sld.cu/galerias/pdf/sitios/ rehabilitacion-adulto/alteraciones de la atencion tras dano.pdf

Sohlberg, M. y Mateer, C. (2001). Cognitive rehabilitation: An integrative neuropsychological approach: Introduction to cognitive rehabilitation. New York: The Guilfords Press.

Organización de las Naciones Unidas para la educación, la ciencia y la cultura. (2013). Directrices para las políticas de aprendizaje móvil.

Recuperado de: https://unesdoc.unesco.org/in/documentViewer.xhtml? $\mathrm{v}=2.1 .196 \mathrm{\& id}=\mathrm{p}::$ usmarcdef $0000219662 \mathrm{ffile}=/ \mathrm{in} / \mathrm{rest} / \mathrm{annotationSVC} /$ DownloadWatermarkedAttachment/attach import ldf6e042-49f5-4626-97525692503f8477\%3F \%3D219662spa.pdf\&updateUrl=updateUrl7142\&ark=l ark:/48223/pf0000219662/PDF/219662spa.pdf.multi\&fullScreen=true\&locale $=$ es\#114 13 ES ED int.indd\%3A.4997

Villaroig, L., Muiños, M. (2017). La Atención: Principales rasgos, tipos y estudio. Trabajo final de grado maestro/a de educación infantil/primaria. Universitat Jaume I. España. Recuperado de: http://repositori.uji.es/xmlui/ bitstream/handle/10234/177765/TFG 2018 VillarroigClaramonte Laura. pdf?sequence $=1$ \&is Allowed $=\mathrm{y}$ 